\title{
Stem cells and neurogenesis: Implications in the pathogenesis and Treatment of Alzheimer's Disease
}

\author{
Winnie $\mathbf{N g}$ (University of Toronto) \\ News Reporter (HSI 2012-2013)
}

Alzheimer's disease (AD) is the most common form of dementia and is primarily characterized by learning, memory, and cognitive loss due to initial neuronal cell death in the hippocampus that spreads to other areas of the cerebral cortex and is eventually fatal. $A D$ is progressive and irreversible. Presently there is no cure, and many of the available treatments only aim to modify the course of disease. According to the Alzheimer Society of Canada, dementia currently affects approximately 500,000 Canadians; however, this number is projected to more than double by 2038. The economic burden of dementia in Canada was upwards of 8 billion dollars in direct health costs in 2008,

\section{"[M]ore research is needed}

to develop and test novel treatments that may mitigate its effects or lead to the development of a cure." with an additional 1.8 billion dollars for indirect costs such as lost wages and decreases in the labour productivity of patients and caregivers. ${ }^{1}$ Moreover, this does not account for the physical and emotional burden of $A D$ for family members and other caregivers who witness the effects of cognitive decline. Clearly, effective strategies are needed that support all individuals affected by $A D$, and significantly more research is needed to develop and test novel treatments that may mitigate its effects or lead to the development of a cure.

Age is one of the most important risk factors for $A D$, although $A D$ is not necessarily a disease of ageing, as previously believed. It was over a century ago that Dr. Alois Alzheimer found plaques and tangles in the brain of a patient with 'senile dementia' and these markers have become hallmarks for the diagnosis of AD. ${ }^{2}$ Plaques refers to beta amyloid plaques that are comprised of beta amyloid protein $(A B)$ produced from the cleavage of amyloid precursor protein (APP), whereas tangles refers to neurofibrillary tangles that form due to tau protein aggregates. ${ }^{3}$ However,

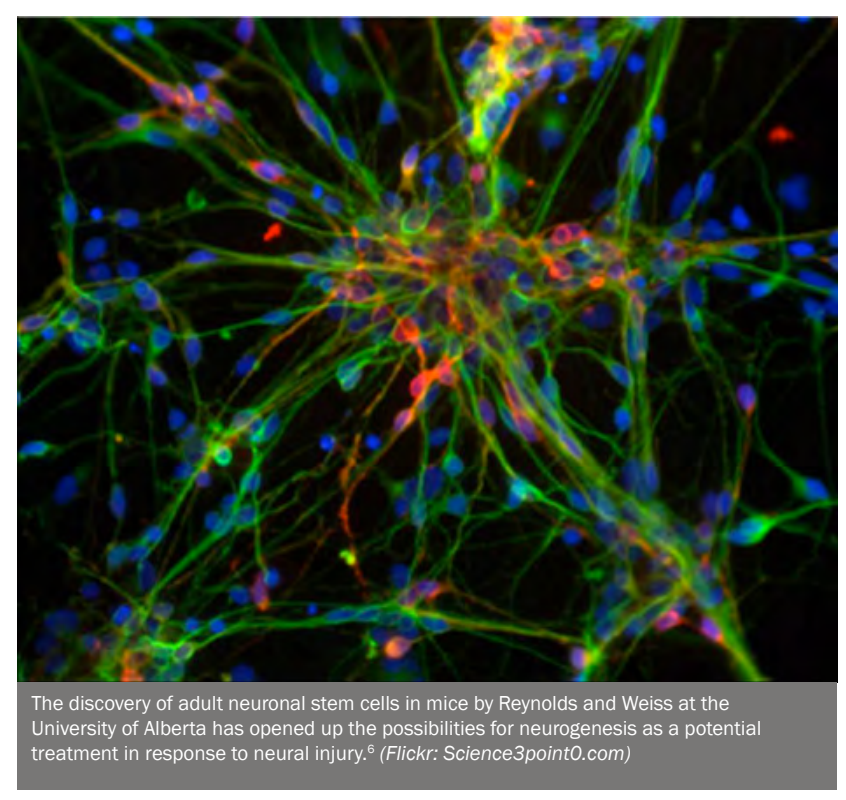

beta amyloid protein and tau protein have been observed in humans post-mortem without AD dementia, ${ }^{4}$ indicating that these proteins may be necessary but insufficient to induce AD. Nevertheless, most AD research has focused on investigating the pathogenesis of $A D$ in the context of the plaques and tangles.

Stem cells have become a very exciting area of regenerative medicine since the discovery of human embryonic stem cells in $1998 .{ }^{5}$ The inherent characteristics of stem cells, having a high capacity for self renewal and the ability to differentiate into various cell types, provides the potential for a unique opportunity to treat diseases that require regeneration or replacement of damaged cellular tissue. Neurons were primarily considered a terminally differentiated cell type, meaning that these specialized cells could not regenerate themselves. However, the discovery of adult neuronal stem cells in mice by Reynolds and Weiss at the University of Alberta has opened up the possibilities for neurogenesis as a potential treatment in response to neural injury. ${ }^{6}$ More recently, Fernandes et al. discovered that neuronal cells could be generated from skin-derived precursors found in the dermis, ${ }^{7}$ which extends the potential for autologous stem cell transplantation in the treatment of neurological disorders. 
Dr. Karl Fernandes, an assistant professor in the Department of Pathology and Cell Biology at the Universite de Montreal and the Canada Research Chair in Stem Cell Neurobiology, is taking an alternative approach to studying $A D$ and investigating how the biology of stem cells is affected by neurological diseases such as $A D$ and how they could be involved with the pathology. "Neuronal stem cells (NSCs) are involved in all four major areas of neuroscience; including how the brain was built, how it works, what happens when it breaks down, and how to fix it," says Dr. Fernandes, which demonstrates why it is such a good candidate to study in the context of neurological diseases.

Since $A D$ is very difficult to study in humans, animal models of $A D$ have been useful to study the mechanisms of $A D$. One such model is the triple-transgenic mouse model $3 \times \mathrm{Tg}$, which in short is a PS1 ${ }_{\mathrm{M} 146 \mathrm{~V}}$ knock-in mouse that contains the human $\mathrm{APP}_{\text {Swe }}$ and human tau $\mathrm{P}_{\text {P301L }}$ transgenes that results in the development of the hallmark plaques and tangles observed in AD pathology. ${ }^{8}$ Using this model, Dr. Fernandes' research group found that $3 \times \mathrm{Tg}$ mice had decreased neurogenesis in the hippocampus and subventricular zones through BrdU and Ki-67 labelling as compared to the wildtype mice, and this was associated with decreased cognitive function..$^{9}$ Moreover, this down regulation in neurogenesis in the 3xTg mice was similar to what was observed in the wild-type mice at an older age. What is more interesting is that the decrease in neurogenesis in the $3 \times \operatorname{Tg}$ mice preceded the development of the hallmark plaques and tangles of $A D$. These findings are intriguing as it shows changes in the NSC population in an AD-like environment and suggests that the breakdown of neurogenesis may be implicated in

"If activation of stem cells is On the other hand, increased in the hippocampus it these findings do may increase cognitive function as not rule out that $A D$ a whole, independent of whether pathology may lead we get the disease or not." to the decrease in Dr. Fernandes neurogenesis.

It must be cautioned that it is unknown whether the AD pathology in animals is truly representative of what occurs in humans. Thus, it questions the relevancy of animal models. However, in humans only an endpoint snapshot can be obtained of $A D$. There are many animal models of $A D$; this particular $3 \times T g$ mouse model is unique in that it reproduces the $A D$ environment with regards to both the plaques and tangles. Although questions still remain about whether these results are translatable to humans, according to Dr. Fernandes the first step is to see whether these findings can be translated to other AD models. In terms of the big picture, "the implications of this research are that decreased neurogenesis at an earlier time point may accelerate cognitive defects," says Dr. Fernandes and, "if activation of stem cells is increased in the hippocampus it may increase cognitive function as a whole, independent of whether we get the disease or not." Presently neurogenesis cannot be measured in humans, although the technology is getting close. Based on current research, it is more likely that any clinical approach would involve modifying the course of $A D$ rather than developing a cure. Transplantation of NSCS is also, as of yet, unlikely as there are still concerns whether proper neurons and connections will develop or whether NSCs will provide trophic support rather than neuronal replacement. One area of promise may be to stimulate neurogenesis from endogenous NSCs to treat $A D$ using drugs already available on the market that may activate specific pathways required by stem cells for recruitment and/or differentiation; as was found with thediabetes drug metformin, which increased neurogenesis and enhanced cognitive function in mice via the atypical protein kinase C-CBP transcriptional co-activator (aPKC-CBP) pathway. ${ }^{10}$

Perhaps the most profound implications are that studying the biology of NSCs, rather than focusing strictly on its potential as a treatment, may help to understand and even prevent debilitating neurological diseases in the future and many of these findings could be translatable between stem cell fields. Stem cell research has been dominated by Canadian innovation since their discovery by Till and McCullough in the 1960s and foundations like the Stem Cell Network continue to support Canadian researchers to further fuel the enormous potential for stem cells to improve and advance human health.

\section{Acknowledgements}

Special thanks to Dr. Fernandes for his contribution to this news article.

\section{References}

1. Alzheimer Society of Canada. Rising Tide: The Impact of Dementia on Canadian Society. Toronto: Alzhemier Society of Canada; 2010.

2. Citron M. Alzheimer's disease: strategies for disease modification. Nat Rev Drug Dis. 2010; 9(5):387-98.

3. Gotz J, Ittner LM. Animal models of Alzheimer's disease and frontotemporal dementia. Nat Rev Neurosci. 2008; 9(7):532-44.

4. Guillozet AL, Weintraub S, Mash DC, Mesulam M. Neurofibrillary tangles, amyloid, and memory in aging and mild cognitive impairment. Arch Neurol. 2003; 60(5):729-36.

5. Thomson JA, Itskovitz-Eldor J, Shapiro SS, Waknitz MA, Swiergiel JJ, Marshall VS, et al. Embryonic stem cell lines derived from human blastocysts. Science. 1998; 282(5391):1145-47. 


\section{HEALTH SCIENCE INQUiRY}

6. Reynolds BA, Weiss S. Generation of neurons and astrocytes from isolated cells of the adult mammalian central nervous system. Science. 1992; 255(5052):1707-10

7. Fernandes KJL, Kobayashi NR, Gallagher CJ, Barnabe-Heider F, Aumont A, Kaplan DR, et al. Analysis of the neurogenic potential of multipotent skinderived precursors. Exp Neurol. 2006; 201(1):32-48.

8. Oddo S, Caccamo A, Shepherd JD, Murphy MP, Golde TE, Kayed R, et al. Triple-transgenic model of Alzheimer's Disease with plaques and tangles: intracellular Ab and synaptic dysfunction. Neuron. 2003; 39(3):409-21.
9. Hamilton LK, Aumont A, Julien C, Vadnais A, Calon F, Fernandes KJL. Widespread deficits in adult neurogenesis precede plaque and tangle formation in the 3xTg mouse model of Alzheimer's disease. Eur J Neurosci. 2010; 32(6):905-20.

10. Wang J, Gallagher D, DeVito LM, Cancino Gonzalo I, Tsui D, He L, et al. Metformin activates an atypical PKC-CBP pathway to promote neurogenesis and enhance spatial memory formation. Cell Stem Cell. 2012; 11(1):23-35.

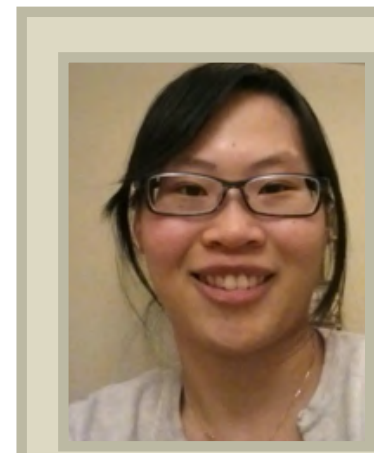

\section{Winnie $\mathrm{Ng}$}

Winnie Ng completed her B.Sc. in Biomedical Toxicology at the University of Guelph and is currently finishing her Ph.D. at the University of Toronto in the Department of Pharmaceutical Sciences. Her thesis research focuses on the mechanisms of idiosyncratic drug reactions and involves testing the immunological changes induced by aromatic amine drugs in animal models. Upon completion of her doctorate degree, she hopes to maintain a similar path and pursue a career in drug safety. She is also actively involved in the Life Sciences Career Development Society and enjoys teaching others about science as a Let's Talk Science volunteer. In her spare time she likes to be outdoors exploring new trails, travelling, learning about new cultures, and she is also fascinated with the Group of Seven artwork. 